\title{
Equação de biomassa e estoque de carbono do pinhão manso, no município de Viçosa, MG
}

\author{
Biomass equation and carbon stock of jatropha crop, in Viçosa, Minas Gerais, Brazil
}

\author{
Diego de Paula Toledo ${ }^{* *}$ Laércio Antônio Gonçalves Jacovine ${ }^{\mathrm{I}}$ \\ Carlos Moreira Miquelino Eleto Torres ${ }^{\mathrm{I}}$ Carlos Pedro Boechat Soares ${ }^{\mathrm{I}}$
}

\section{RESUMO}

O presente trabalho teve como objetivo estimar equações de biomassa e o estoque de carbono da cultura do Pinhão Manso (Jatrophacurcas L.). A área estudada localizase no município de Viçosa, MG. O plantio foi realizado em espaçamento 3,5x3,0m, em 4,64ha. Para quantificação da biomassa, foi utilizado o método direto e destrutivo, aplicado às árvores-amostra, que foram selecionadas de acordo com as medidas da altura, do diâmetro das copas e do número de ramos. A determinação da biomassa de cada árvore foi obtida pelo método da proporcionalidade. Os modelos testados foram adaptados de Spurr e de Schumacher \& Hall, para biomassa aérea e biomassa total (biomassa aérea mais biomassa de raízes pivotantes). O estoque de carbono foi estimado através da multiplicação da biomassa seca pelo teor de carbono da matéria seca, que foi obtido pelo método da calcinação em mufla. A estimativa do $\mathrm{CO}_{2}$ equivalente estocado foi obtida pela multiplicação do estoque de carbono pelo fator 44/12. A equação que obteve melhor ajuste e que foi utilizada para determinação do estoque de carbono deste estudo foi a de Spurr, com os dados de biomassa total, $B=0,7601^{*}\left(D C^{2 *} H\right)^{0,8949}$, em que $B=$ biomassa $(\mathrm{kg}) ; \mathrm{DC}=$ diâmetro de copa $(\mathrm{m})$; e $H=$ altura (m). O estoque de carbono encontrado da cultura, no quarto ano, foi de $6,79 \mathrm{MgC} \mathrm{ha}{ }^{-1}$, correspondendo a $24,89 \mathrm{Mg}$ $\mathrm{CO}_{2(\text { eq }} h \mathrm{ha}^{-1}$. Os resultados mostram que o pinhão-manso é ambientalmente viável para elaboração de projetos $M D L$ de florestamento/reflorestamento ou em projetos de carbono para mercados voluntários, agregando renda ao produtor rural e melhorando a atratividade financeira da cultura.

Palavras-chave: mecanismo de desenvolvimento limpo, protocolo de Kyoto, biodiesel, Jatrophacurcas $L$.

\begin{abstract}
This study had the objectives to estimate equations of biomass and carbon stock of Jatropha (Jatrophacurcas L.). The area of this study is located in Viçosa, Minas Gerais, planting carried out in $3.5 \times 3.0 \mathrm{~m}$ spacing at $4.64 \mathrm{ha}$. To biomass quantification, we used the direct destructive method, applied to trees, which were selected according to the measurements of height, canopy diameter and number of branches. The tree biomass determination was obtained by the proportionality method. The adapted models were from Spurr and Schumacher \& Hall, for biomass and total biomass (aboveground biomass more taproots biomass). The carbon stock was estimated multiplying the biomass by the carbon content of dry matter, which was obtained by calcination in a muffle. The estimate of the equivalent $\mathrm{CO}_{2}$ stored was obtained by multiplying the carbon stock by a factor 44/12. The equation with better model and who was used for carbon stock determination was to Spurr, using total biomass data, $B=0.7601^{*}\left(C D^{2 *} H\right)^{0.8949}$, where $B=$ biomass $(\mathrm{kg})$; $C D=$ canopy diameter $(\mathrm{m})$; e $H=$ height $(\mathrm{m})$. The carbon stock of culture, founded to the fourth year, was

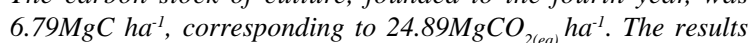
shows that Jatropha is environmentally viable for development of CDM afforestation / reforestation projects or carbon projects to voluntary markets, adding income to farmers and improving the financial attractiveness of the crop.
\end{abstract}

Key words: clean development mechanism, Kyoto protocol, biodiesel, Jatrophacurcas $L$.

\section{INTRODUÇÃO}

Com o aumento da concentração de gases na atmosfera, o efeito estufa vem se agravando e

'Departamento de Engenharia Florestal (DEF), Universidade Federal de Viçosa (UFV), Avenida P. H. Rolfs, s/n, Campus UFV, 36570-000, Viçosa, MG, Brasil. E-mail: diegoptoledo@yahoo.com.br. *Autor para correspondência. 
ocasionando a elevação da temperatura global. Amelhor estimativa da mudança de temperatura da superfície terrestre é de $0,76^{\circ} \mathrm{C}$ de acréscimo, desde o século XIX (IPCC, 2007). Modelos climáticos preveem que a temperatura global irá aumentar de 1,4 a $5,8^{\circ} \mathrm{C}$ até 2100 . Essas alterações deverão ser as maiores mudanças climáticas experimentadas nos últimos 10.000 anos (UNEP \& UNFCCC, 2002). As principais fontes dos gases de efeito estufa (GEE) é a queima de combustíveis fósseis e o desmatamento.

Devido à evidência do aumento da emissão dos GEE e ao aquecimento global, a preocupação com o clima vem ganhando importância mundial. Inúmeras reuniões internacionais vêm ocorrendo, na intenção de estabelecer políticas públicas que tragam possíveis soluções para evitar ou, pelo menos, reduzir a emissão desses gases.

Dos mecanismos de flexibilização, o Mecanismo de Desenvolvimento Limpo (MDL) é o único que permite a participação de países em desenvolvimento, como o Brasil. O propósito do MDL é contribuir com os países em desenvolvimento (Partes não Anexo, UNFCCC, 2011), para que viabilizem projetos que promovam o desenvolvimento sustentável e contribuam para o objetivo final do Protocolo de Kyoto, prestando assistência aos países Partes Anexo I (UNFCCC, 2011), para que estes cumpram suas metas de redução de GEE (LOPES, 2002).

Conforme descrito nas ferramentas metodológicas para projetos de florestamento e reflorestamento do Protocolo de Kyoto, é previsto o uso de modelos matemáticos que utilizam variáveis dendrométricas para a quantificação dos estoques de carbono nos compartimentos arbóreos (UNFCCC, 2011).

Com isso, a utilização do pinhão manso como matéria-prima da indústria do biodiesel pode tornar-se elegível como uma atividade de MDL, tanto pela absorção de carbono por parte da cultura quanto da utilização de suas sementes para produção de energia renovável, em detrimento do uso de combustíveis fósseis. Diante disso, o objetivo deste trabalho foi estimar equações de biomassa e quantificar o estoque de carbono da cultura do pinhão manso.

\section{MATERIAL E MÉTODOS}

A área de estudo está localizada no município de Viçosa - MG, localizada nas coordenadas geográficas $20^{\circ} 48^{\prime}$ 'S e $42^{\circ} 52^{\prime} \mathrm{W}$ (sistema de projeção cilíndrica, DATUM: SAD 69), altitude média de 700m. A área de efetivo plantio é de 4,52ha. A execução do plantio se deu em janeiro de 2006 no espaçamento $3,5 \times 3,0 \mathrm{~m}$, sendo plantadas 4.300 mudas. Segundo a classificação de Köeppen, o clima na região é Cwb, com chuva mal distribuída ao longo do ano, verão chuvoso e inverno seco. O total pluviométrico anual médio é de $1.221 \mathrm{~mm}$ e a temperatura média é de $19^{\circ} \mathrm{C}$ (VIANELLO et al., 1991).

Com base na moda do número de ramos e nas médias da altura e do diâmetro de copa, foram selecionadas, por ano, oito árvores-amostra para quantificação da biomassa dos componentes da parte aérea (folhas e ramos) e, dessas oito, escolheu-se aleatoriamente quatro árvores-amostra para a quantificação da biomassa do sistema radicular (raiz pivotante). A determinação da biomassa foi realizada pelo método direto e destrutivo de árvores (TEIXEIRA et al., 1994), sendo que, devido à caducifolia da espécie, o material foi coletado entre os meses de dezembro e março, com intervalos próximos a 12 meses entre as coletas e o início das coletas em março de 2007. A determinação da biomassa seca da árvore individual foi obtida através do método da proporcionalidade (TEIXEIRA et al., 1994;SOARES et al., 1996).

Para a estimativa das equações de biomassa, foram adaptadas as equações volumétricas de Spurr (SPURR, 1952) e Schumacher \& Hall (SCHUMACHER \& HALL, 1933) (Tabela 1). Além do cálculo das medidas

Tabela 1 - Equações volumétricas adaptadas de Spurr e Schumacher \& Hall usadas no ajuste de equação de biomassa para árvore individual de JatrophacurcasL.

\begin{tabular}{ll}
\hline Equação & Adaptado \\
\hline $\mathbf{B}=\boldsymbol{\beta}_{0} *\left(\mathbf{D C}^{2} * \mathbf{H}\right)^{\boldsymbol{\beta}_{1}}+\boldsymbol{\varepsilon}$ & Spurr \\
$\mathbf{L n B}=\boldsymbol{\beta}_{0}+\boldsymbol{\beta}_{1} * \operatorname{LnDC}+\boldsymbol{\beta}_{2} * \operatorname{Ln} \mathbf{H}+\boldsymbol{\varepsilon}$ & Schumacher \& \\
\hline
\end{tabular}

$\mathrm{B}=$ biomassa; $\mathrm{DC}=$ diâmetro de copa $(\mathrm{m}) ; \mathrm{H}=$ Altura $(\mathrm{m}) ; \beta 0$, $\beta 1, \beta 2=$ parâmetros do modelo; $\mathrm{Ln}=$ logaritmo natural; $\epsilon=$ erro aleatório. 
de precisão $\left(\mathrm{R}^{2}, \mathrm{~S}_{\mathrm{yx}}\right.$ e $\left.\mathrm{CV}\right)$ das equações, foi analisado o gráfico de dispersão dos resíduos para determinar qual equação melhor representava a biomassa individual das árvores.

A biomassa total por hectare de cada ano foi estimada proporcionalmente à área representada pela amostragem das árvores, sendo obtida pela multiplicação do número de árvores amostradas no ano pela área útil de cada planta, segundo o espaçamento utilizado pela cultura $\left(3,5 \times 3,0 \mathrm{~m}\right.$, área útil por planta $\left.10,5 \mathrm{~m}^{2}\right)$. O teor de carbono da matéria seca foi calculado pela média de 3 amostras de cada compartimento da planta (folhas, ramos e raiz pivotante) obtidas no quarto ano.

O teor de carbono de cada amostra foi determinado pelo método da calcinação em mufla, segundo metodologia adotada pelo Laboratório de Solos Florestais da Universidade Federal de Viçosa, em que cada amostra de $1 \mathrm{~g}$ foi colocada em um cadinho de porcelana sem tampa, e levada à mufla na temperatura de $550^{\circ} \mathrm{C}$, por três horas, até completa calcinação. A amostra foi retirada da mufla e resfriada em dissecador para posterior pesagem. O teor de carbono foi calculado pela seguinte equação:

$\mathrm{CT}=(\mathrm{Ms} / \mathrm{Mr}) * 100$, em que: $\mathrm{CT}=$ Teor de carbono, em \%; Ms = massa do resíduo, em g; $\mathrm{Mr}=$ massa da amostra seca, em g.

Em seguida, o estoque de carbono da planta foi obtido por média ponderada de cada compartimento e, posteriormente, extrapolado para toneladas por hectare. Pelo fato de as negociações no mercado de créditos de carbono serem efetuadas em função do $\mathrm{CO}_{2}$ equivalente $\left(\mathrm{CO}_{2(\mathrm{eq})}\right)$, tornou-se necessária a conversão do carbono em $\mathrm{CO}_{2}$. Como descrito pelo IPCC (2003), utilizou-se uma taxa de conversão de 44/ 12, multiplicada pelo estoque de carbono, que corresponde ao peso atômico do $\mathrm{CO}_{2}$ (44), dividido pelo peso atômico do carbono (12).

\section{RESULTADOS E DISCUSSÃO}

Os coeficientes de determinação $\left(\mathrm{R}^{2}\right)$ das equações de biomassa aérea e total, referentes tanto às equações de Schumacher \& Hall quanto às de Spurr, foram satisfatórios. Contudo, nas equações de Schumacher \& Hall, os parâmetros $\beta_{0}$ e $\beta_{1}$ para biomassa total e $\beta_{1}$ para biomassa aérea não foram estatisticamente significativos e comprometeram o ajuste dessas equações. Em ambas as equações de Spurros, os parâmetros testados foram estatisticamente significativos (Tabelas 2 e 3 ).

Os gráficos de dispersão dos resíduos dos valores observados e estimados das equações de Spurr, tanto da altura quanto do diâmetro de copa, mostram tendência de subestimar a biomassa em diâmetros de copa inferiores a $1,50 \mathrm{~m}$ e alturas inferiores a 2,00m. Esse fato pode ser explicado pela adubação de plantio

Tabela 2 - Estimativa dos parâmetros, erro-padrão e significância dos parâmetros da equação adaptada de Schumacher \& Hall para Biomassa aérea e Biomassa total de árvore individual de Jatrophacurcas L.

\begin{tabular}{|c|c|c|c|}
\hline Coeficientes & Estimativa & Erro-Padrão da estimativa & $\mathrm{p}$ \\
\hline$\widehat{B_{0}}$ & -1.0115 & $\begin{array}{r}\text { omassa aérea----- } \\
0.2808\end{array}$ & $1,2 \times 10^{-3 * *}$ \\
\hline $\begin{array}{l}\overrightarrow{\beta_{0}} \\
\overrightarrow{\beta_{1}}\end{array}$ & 0,0790 & $\begin{array}{l}0,2000 \\
0,4094\end{array}$ & 0,8483 NS \\
\hline$\widehat{\beta_{2}}$ & 2,8489 & 0,4885 & $2,5 \times 10^{-6^{* *}}$ \\
\hline $\mathrm{R}^{2}$ & 0,8733 & & \\
\hline CV $(\%)$ & 29,97 & & \\
\hline$\widehat{\beta_{0}}$ & $-0,4461$ & 0,4307 & $0,3192^{\mathrm{NS}}$ \\
\hline$\widehat{\beta_{1}}$ & 0,4392 & 0,6442 & $0,5073^{\mathrm{NS}}$ \\
\hline $\overrightarrow{\beta_{2}}$ & 2,3182 & 0,7704 & $0,0101^{* *}$ \\
\hline $\mathrm{R}^{2}$ & 0,8619 & & \\
\hline CV (\%) & 32,24 & & \\
\hline
\end{tabular}

** = significante pelo teste "t" (Student) a 5\% de probabilidade; NS = não significativo; $\mathrm{R}^{2}=$ coeficiente de determinação; CV = coeficiente de variação.

Ciência Rural, v.42, n.11, nov, 2012. 
Tabela 3 - Estimativa dos parâmetros, erro-padrão e significância dos parâmetros da equação adaptada de Spurr para Biomassa aérea e Biomassa total de árvore individual de Jatrophacurcas L.

\begin{tabular}{|c|c|c|c|}
\hline Coeficientes & Estimativa & Erro-Padrão da estimativa & $\mathrm{p}$ \\
\hline $\overrightarrow{\beta_{0}}$ & 0,4973 & 0,1252 & $4,1 \times 10^{-4 * *}$ \\
\hline $\overrightarrow{\beta_{1}}$ & 0,9408 & 0,0789 & $6,5 \times 10^{-13} * *$ \\
\hline $\mathrm{R}^{2}$ & 0,8533 & & \\
\hline CV $(\%)$ & 44,36 & & \\
\hline $\overrightarrow{\beta_{0}}$ & 0,7601 & 0,2703 & $1,4 \times 10^{-2} * *$ \\
\hline $\overrightarrow{\beta_{1}}$ & 0,8949 & 0,1117 & $1,3 \times 10^{-6 * *}$ \\
\hline $\mathrm{R}^{2}$ & 0,8562 & & \\
\hline CV $(\%)$ & 42,71 & & \\
\hline
\end{tabular}

** = significante pelo teste "t" (Student) a 5\% de probabilidade; NS = não significativo; $\mathrm{R}^{2}=$ coeficiente de determinação; CV = coeficiente de variação.

praticada pelo produtor que proporcionou maior acúmulo de biomassa no primeiro ano da cultura. Após esses valores, observa-se distribuição mais uniforme dos resíduos (Figura 1), melhor representando a tendência de crescimento da biomassa em função da altura e diâmetro de copa. Assim, optou-se pelo uso da equação de Spurr, com os dados de biomassa total para as estimativas de carbono, por esta apresentar melhor ajuste:

$\mathrm{B}=0,7601 *\left(\mathrm{DC}^{2 *} \mathrm{H}\right)^{0,8949}$, em que $\mathrm{B}=$ biomassa (kg); DC = diâmetro de copa (m); e H = altura (m).

A biomassa média por parte da planta foi maior nos ramos, seguidos pelas raízes e folhas, em todas as idades observadas (Figura 2). Essas medidas podem ser explicadas pelo fato de os tecidos do caule apresentarem maior acúmulo de celulose, hemiceluloses e ligninas nas paredes de suas células, já que este tecido apresenta função estrutural na planta (TAIZ \& ZEIGER, 2004).

O teor de carbono das folhas foi de $48,96 \pm 0,25 \% \mathrm{C}$, nos ramos de $51,07 \pm 0,48 \% \mathrm{C}$ e nas raízes de $46,70 \pm 0,86 \% \mathrm{C}$. Esses valores estão compatíveis com o teor de carbono em biomassa arbórea seca de 50\%, indicado pelo IPCC (2003). Contudo, se utilizado o valor default de $50 \%$ para o teor de carbono, proposto pelo IPCC (2003), as estimativas de estoque de carbono para o pinhão manso seriam superestimadas.

$\mathrm{O}$ estoque total de carbono da área no quarto ano de cultura de $6,79 \mathrm{MgC} \mathrm{ha}^{-1}$, que corresponde a $24,89 \mathrm{MgCO}_{2(\mathrm{eq})} \mathrm{ha}^{-1}$, foi superior ao estoque de carbono de uma pastagem de $0,42 \mathrm{MgC} \mathrm{ha}^{-1}$, observado por RIBEIRO et al. (2010), mostrando que a cultura do Pinhão Manso pode tornar-se um projeto ambientalmente viável de MDL (Tabela 4). Convertendo os resultados do estudo de CALDEIRA (2000) de biomassa de três procedências de acácia negra (Acaciamearnsii De Wild.) com 2,4 anos de idade em Butiá-RS, tem-se para as procedências BatemansBay, Bodalla e Lake George Bunge Dore estoques de carbono de, respectivamente, 18,05; 9,52 e 20,55MgC $\mathrm{ha}^{-1}$. Esses dados corroboram o potencial de estocagem de carbono do pinhão manso.

Ao quarto ano, a cultura estudada apresenta um incremento médio anual (IMA), em carbono, equivalente, de $6,64 \mathrm{MgCO}_{2(\mathrm{eq})} \mathrm{ha}^{-1}$.ano ${ }^{-1}$. Esse incremento é superior ao IMA de $3,19 \mathrm{MgCO}_{2(\mathrm{eq})} \mathrm{ha}^{-1}$ ano $^{-1}$ para cultura do cacaueiro, aos 6 anos de idade, e compatível com o incremento de $9,16 \mathrm{MgCO}_{2(\mathrm{eq})} \mathrm{ha}^{-1} \mathrm{ano}^{-1}$ para a cultura da seringueira, aos 34 anos de idade, observados por COTTA et al. (2008) no consórcio seringueira-cacau.

\section{CONCLUSÃO}

A equação de Spurr, utilizada para estimar a biomassa total por árvore individual de Jatrophacurcas L., pode ser utilizada como referência em outros estudos

Ciência Rural, v.42, n.11, nov, 2012. 


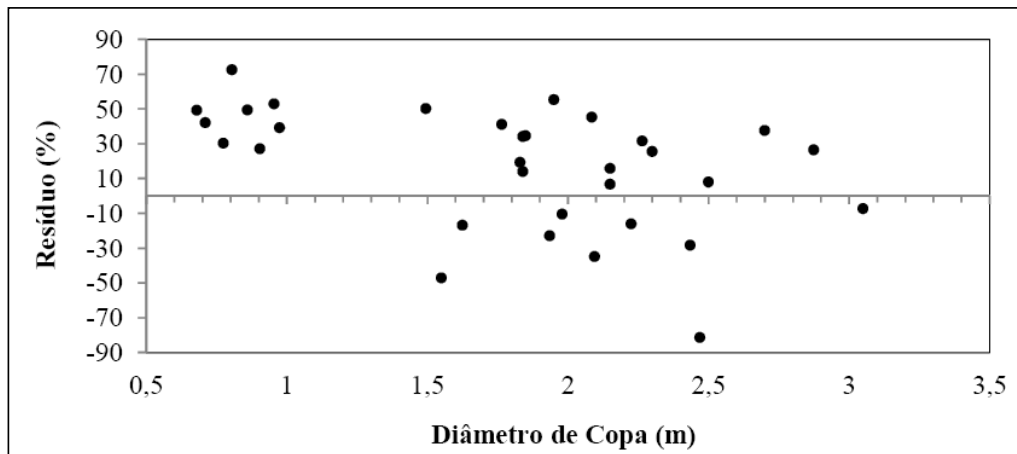

(A)

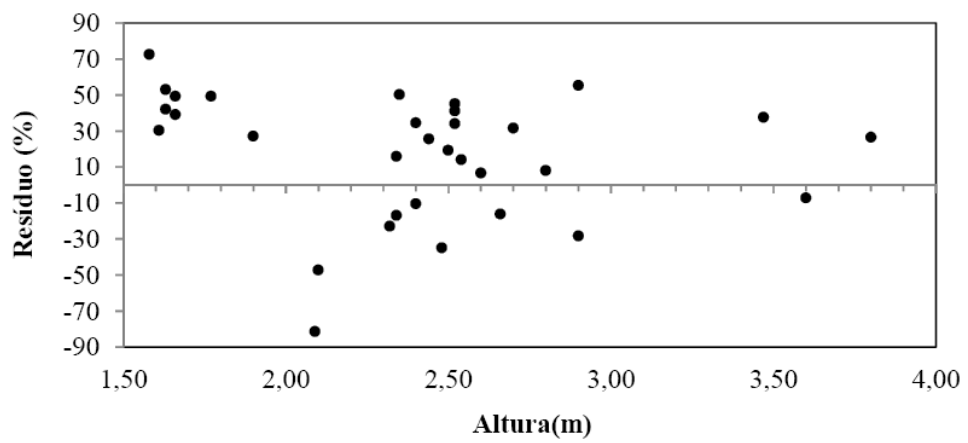

(B)

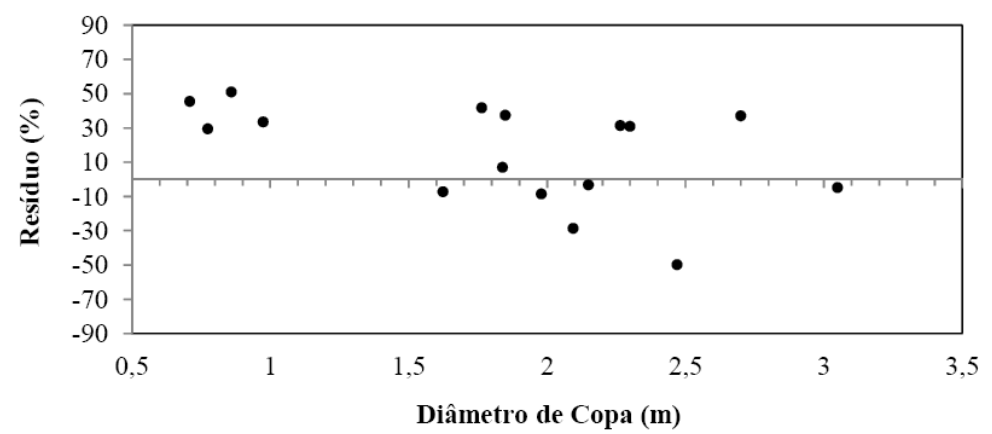

(C)

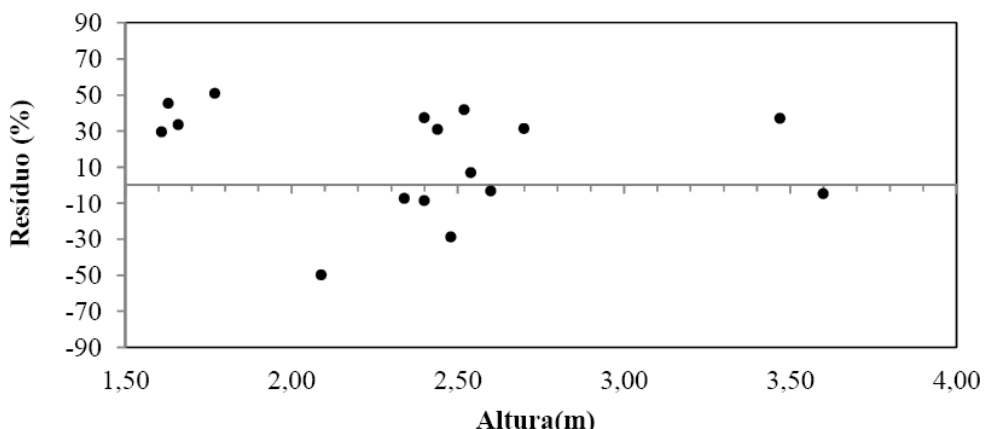

(D)

Figura 1 - Dispersão dos resíduos da equação de Spurr para Biomassa aérea por árvore, em relação ao diâmetro de copa (A) e a altura (B); e para Biomassa total por árvore, em relação ao diâmetro de copa $(\mathrm{C})$ e à altura (D) de Jatrophacurcas L. 


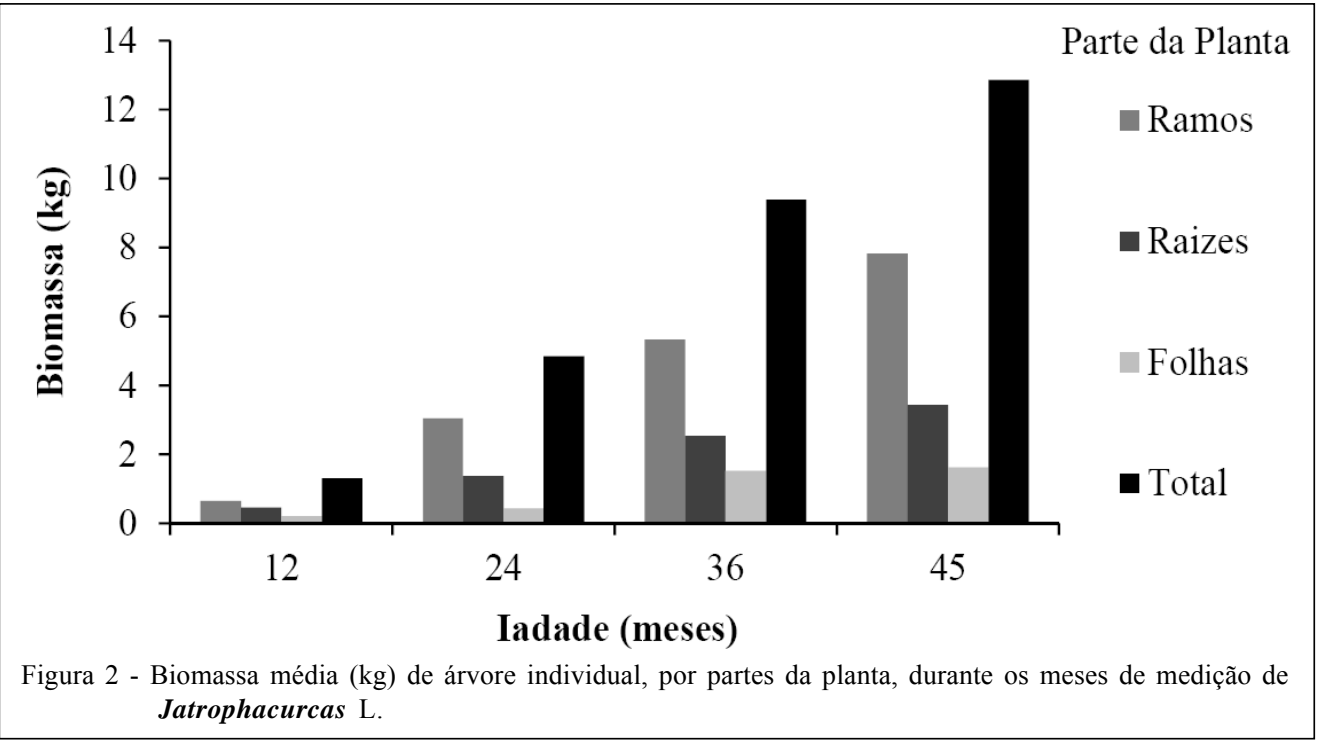

que visem a obter tais dados. O ajuste da equação é satisfatório, devendo ser utilizado com ressalva em diâmetros de copa inferiores a 1,50m e alturas inferiores a 2,00m, pela tendência de subestimação da biomassa, que geralmente ocorre no primeiro ano da cultura.

$O$ pinhão manso mostra-se atrativo ambientalmente para pleitear créditos de carbono através de projetos de MDL. As estimativas de biomassa e estoque de carbono de $6,79 \mathrm{MgC} \mathrm{ha}^{-1}$, encontrados neste estudo, servem de base para trabalhos de elegibilidade da cultura em projetos de MDL ou em mercados voluntários.

\section{AGRADECIMENTOS}

Os autores gostariam de agradecer ao proprietário Paulo Afonso, por disponibilizar a realização deste estudo em sua propriedade. Ao Conselho Nacional de Pesquisa e Desenvolvimento Científico e Tecnológico (CNPq), pela concessão de financiamento e bolsas de pesquisa.

Tabela 4 - Estoques de carbono (C) e carbono equivalente $\left(\mathrm{CO}_{2(\mathrm{eq})}\right)$ da cultura de Jatrophacurcas L. ao longo dos meses, no município de Viçosa - MG.

\begin{tabular}{lcc}
\hline Idade (meses) & $\mathrm{C}\left(\mathrm{MgC} \mathrm{ha}^{-1}\right)$ & $\mathrm{CO}_{2(\mathrm{eq})}\left(\mathrm{MgCO}_{2(\mathrm{eq})} \mathrm{ha}^{-1}\right)$ \\
\hline 12 & 0,63 & 2,30 \\
24 & 3,21 & 11,78 \\
36 & 3,81 & 13,96 \\
45 & 6,79 & 24,89 \\
\hline
\end{tabular}

\section{REFERÊNCIAS}

CALDEIRA, M.V.W. Quantificação da biomassa e do conteúdo de nutrientes em diferentes procedências de acácia-negra (Acacia mearnsii De Wild.). Ciência Rural, v.30, n.1, p.195196, mar, 2000. Disponível em: <http://www.scielo.br/ s c i e lo.php? s cript $=$ sci arttext\&pid=S0103$84782000000100031 \& \operatorname{lng}=$ pt\&nrm=iso $>$. Acesso em:10 maio, 2010. doi: 10.1590/S0103-84782000000100031.

COTTA, M.K. et al. Quantificação de biomassa e geração de certificados de emissões reduzidas no consórcio seringueira-cacau. Revista Árvore, v.32, n.6, p.969-978, dez, 2008. Disponível em: $<$ http://www.scielo.br/scielo.php?script $=$ sci arttext\&pid $=\mathrm{S} 0100$ $67622008000600002 \& \operatorname{lng}=$ pt\&nrm=iso $>$. Acesso em: 10 jul. 2010. doi: $10.1590 / \mathrm{S} 0100-67622008000600002$.

IPCC. Climate change 2007: synthesis report. Reports, 2007. Acesso em: 20 maio, 2010. Online. Disponível em: <http:// www.ipcc.ch/pdf/assessment-report/ar4/syr/ar4 syr topic1.pdf $>$.

IPCC. Good practice guidance for land use, land-use change and forestry 2003. Publications, 2003. Acesso em: 20 maio, 2010. Online. Disponível em: $<$ http://www.ipccggip.iges.or.jp/ public/gpglulucf/gpglulucf_contents.html $>$.

LOPES, I.V. (Coord.). O mecanismo de desenvolvimento limpo - MDL: guia de orientação. Rio de Janeiro: Fundação Getúlio Vargas, 2002. 90p.

RIBEIRO, S.C.et al. Quantificação de biomassa e estimativa de estoque de carbono em uma capoeira da Zona da Mata Mineira. Revista Árvore, v.34, n.3, p.495-504, jun, 2010. Disponível em: $<$ http://www.scielo.br/scielo.php?script=sci_arttext\&pid=S010067622010000300013\&lng=pt\&nrm=iso $>$. Acesso em: 10 jun. 2010. doi: 10.1590/S0100-67622010000300013.

SCHUMACHER, F.X.; HALL, F.S. Logarithmic expression of timber-tree volume. Journal of Agricultural Research, v. 47, n.9, p. $719-734,1933$

SOARES, C.P.B et al. Modelos para estimar a biomassa da parte aérea em um povoamento de Eucalyptus grandis na região 
de Viçosa, Minas Gerais. Revista Árvore, v.20, n.2, p.179189,1996

SPURR, S.H. Forest inventory. New York: The Ronald, 1952. 476p.

TAIZ, L.; ZEIGER, E. Fisiologia vegetal. Porto Alegre: Artamed,2004. 719p.

TEIXEIRA, L.B et al. Biomassa vegetal em agroecossistemas de seringueira consorciada com cacaueiro no Nordeste Paranaense. Belém: EMBRAPA CPATU, 1994. 15p. (Boletim de Pesquisa, 153).
UNEP; UNFCCC. Climate change: information kit. Publications, 2002. Acesso em: 20 maio, 2010. Online. Disponível em: <http:/ /unfccc.int/essential_background/background_publications_htmlpdf/ climate_change_information_kit/items/305.php>.

UNFCCC. Estimation of carbon stocks and change in carbon stocks of trees and shrubs in $\mathrm{A} / \mathrm{R} \mathrm{CDM}$ project activities. Methodological tools, 2011. Acesso em: 07 jun. 2011. Online. Disponível em: <http://cdm.unfccc.int/methodologies/ ARmethodologies/tools/ar-am-tool-14-v2.1.0.pdf>.

VIANELLO, R.L. et al. Meteorologia básica e aplicações. Viçosa: UFV, 1991. 449p. 